\title{
Effets d'application sur le long terme de fertilisants organiques et minéraux sur l'agrégation et les activités microbiennes d'un sol tropical sableux au Burkina Faso
}

\author{
Saïdou Nourou SALL ${ }^{(1)}$, Edmond HIEN ${ }^{(2)}$, Aliou GUISSE ${ }^{(3)}$ \\ ${ }^{1}$ Université Gaston Berger, UFR des Sciences Agronomiques, de l'Aquaculture et des Technologies Alimentaires, BP \\ 234, Saint-Louis, Sénégal \\ Université de Ouagadougou, UFR/SVT, 03 BP 7021 Ouagadougou 03, Burkina Faso \\ ${ }^{3}$ Département de Végétale, Faculté des Sciences et Techniques, Université Cheikh Anta Diop, BP. 5005 Dakar- \\ Fann, Sénégal.
}

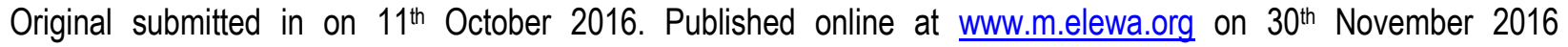
http://dx.doi.org/10.4314/iab.v107i1.3

\section{RÉSUMÉ}

Objectif : L'objectif a été d'étudier l'effet d'une application sur le long terme d'intrants organiques et minéraux sur la formation d'agrégats stables et les activités microbiennes d'un sol tropical sableux du Burkina Faso.

Méthodologie et résultats : Ce travail a été réalisé dans l'observatoire de longue durée de la station agronomique de Saria au Burkina Faso. Dans cet essai, les parcelles en monoculture de sorgho (Sorghum bicolor) ont été soumises à des apports ou non d'intrants organiques (Fumier à $10 \mathrm{t} \mathrm{ha}^{-1} \mathrm{an}^{-1}$ et Paille $83 \mathrm{t}$. $\mathrm{ha}^{-1} \mathrm{an}^{-1}$ ) et minéraux (Urée à $60 \mathrm{~kg} \mathrm{~N}$. ha ${ }^{-1} \mathrm{an}^{-1}$ ) pendant 27 années. Les teneurs en $\mathrm{C}, \mathrm{N}$ et $\mathrm{P}$, les agrégats formés, le potentiel de respiration du sol, les activités de la chitinase et la longueur des hyphes fongiques ont été déterminés. Les résultats ont montré que l'apport sur le long terme des résidus organiques entraine une augmentation de la quantité des macro-agrégats (> $2000 \mu \mathrm{m}$ ) comparé au sol témoin. Cependant, cette augmentation ne dépend pas de la qualité des résidus organiques. Les résultats montrent que les macro-agrégats > $2000 \mu \mathrm{m}$ sont corrélés aux hyphes fongiques, mais pas à l'activité chitinase. L'apport de $\mathrm{N}$ minéral exogène entraine une diminution de la macro-agrégation qui résulterait d'une complexation des polyphénols, agents agrégeants dans le processus d'agrégation pour le long terme.

Conclusion et application des résultats : Cette étude à long terme confirme nos premiers résultats obtenu sur le cours terme et qui stipulent que la formation des macro-agrégats ne dépend pas de la qualité de résidus organiques. La mesure des hyphes fongiques semble plus déterminante dans la formation des macro-agrégats à long terme, par opposition aux activités fongiques dans le court terme.

Mots clés: résidus organiques, $\mathrm{N}$ minéral, agrégation, activités fongiques, Lixisol. 
Long-term effect of organic residues and mineral fertilizers on soil aggregation and microbial activities in a tropical sandy soil in Burkina Faso

ABSTRACT

Objective: The objective was to study the effect of the long-term application of organic amendments and inorganic fertilizers on the formation and stability of soil aggregates and microbial activities in a tropical sandy soil in Saria (Burkina Faso). The relationships between aggregation, microbial activities and the amendments were discussed.

Methodology and results: This study was performed in a long-term (27 years) experimental field in Saria (Burkina Faso) with a continuous sorghum (Sorghum bicolor) cropping system. A randomized block experiment comprising organic amendment (unamended control, straw at $83 \mathrm{t} \mathrm{ha}^{-1}$, manure at $10 \mathrm{t} \mathrm{ha}^{-1}$ ) coupled with mineral (no urea, urea at $60 \mathrm{~kg} \mathrm{ha}^{-1}$ ) was sampled. Chemical parameters $(\mathrm{C}, \mathrm{N}$ and $\mathrm{P}$ ), the formation of aggregates, $\mathrm{C}$ mineralization, fungal hyphae length, and chitinase activity were measured in soil. The results showed that there were more macroaggregates $(>2000 \mu \mathrm{m})$ in soils amended with residues. However, the formation of macro-aggregates was not significantly affected by the quality of residues. The results showed that the macro-aggregates were correlated to fungal hyphae, but not to chitinase activity. The addition of exogenous mineral $\mathrm{N}$ reduced the macro-aggregation that would result from complexation of polyphenols that have been considered to be a primary factor in the aggregation process for the long-term experiment.

Conclusions and application of the results: This long-term study confirms our previous results obtained on the short term incubation of soil amended with residues which showed that the formation of macroaggregates was not affected by the quality of residues. The measurement of fungal hyphae seems to be the most pertinent indicator associated with aggregation in a long-term experiment, as opposed to fungal activity in the short-term experiment.

Keywords: residue quality, mineral N, aggregation, fungal activity, lixisol.

\section{INTRODUCTION}

Dans les zones agricoles de l'Afrique subsaharienne, la baisse de la fertilité des sols et de leurs rendements est attribuable en grande partie au déficit en matières organiques (MO) et à la dégradation accélérée de la structure des sols. Cette dégradation des sols a été décrite comme étant l'une des contraintes majeures à l'autonomie et à la sécurité alimentaire (Bationo et al., 2007 ; Fonte et al., 2010). Pour ces sols déstructurés, le labour, l'intensification des systèmes culturaux, la minéralogie des argiles (kalonite à faible CEC) et des $\mathrm{pH}$ défavorables accentuent leur vulnérabilité. La structure est le mode d'assemblage du sol en micro et macroagrégats. Une bonne structure et par conséquent une forte stabilité des macroagrégats est cruciale pour la productivité et la limitation de l'érosion des sols. Elle régule la disponibilité de l'eau et le statut organique. Elle garantit aussi un environnement favorable pour les racines des plantes et pour la circulation de l'oxygène (Brondik \& Lal, 2005 ; Ouattara et al.,
2006). Dans les sols sableux tropicaux, la MO en décomposition est un facteur important de l'agrégation (Fonte et al., 2010). Durant sa décomposition, la MO peut induire une modification des communautés microbiennes, le développement d'hyphes mycéliens et la sécrétion de molécules (exosaccharides) capables d'agréger les particules solides à l'échelle des agrégats (Tisdall \& Oades, 1982 ; Six et al., 2000). Les hyphes consolident la cohésion des microagrégats en macro-agrégats stables dans les sols tropicaux sableux (Rillig et al., 2010). Les champignons sont également capables de produire des exossacharrides qui ont été depuis longtemps désignés comme des agents transitoires de la stabilité structurale (Tisdall \& Oades, 1982). II est reconnu le rôle capital de la biomasse et/ou de l'activité fongiques sur la formation des agrégats des sols sableux agricoles (Bossuyt et al., 2001; Moreno-Espindola et al., 2008). Des travaux ont mis en exergue 
l'importance de la qualité biochimique des apports de résidus organiques en interaction avec l'utilisation des fertilisants minéraux sur la formation et la stabilité des agrégats (Abiven et al. 2009 ; Chivenge et al., 2011). Les résultats sont très contradictoires. Certains résultats ont montré qu'une addition de fertilisants minéraux augmente la minéralisation des résidus organiques (Berg \& Matzner, 1997), alors que d'autres études ont trouvé que la fertilisation azotée réduit la décomposition des résidus (Bremer et al., 1991 ; Sall et al., 2003) par une diminution de l'abondance des espèces fongiques (Donnison et al., 2000). Cependant, rares sont les études qui mettent en relation la qualité des résidus organiques, les effets de la disponibilité de $\mathrm{N}$ minéral sur les activités fongiques et la formation

\section{MATERIEL ET METHODES}

Site : L'étude a été réalisée dans un observatoire de longue durée de la station de recherche agricole de Saria qui est située à $80 \mathrm{~km}$ à l'ouest de Ouagadougou, capitale du Burkina Faso $\left(12^{\circ} 16^{\prime} \mathrm{N}, 2^{\circ} 9^{\prime} \mathrm{W}\right.$; altitude 300 $\mathrm{m})$. Le climat de cette zone est de type nord-soudanien, caractérisé par deux saisons contrastées. La pluviométrie annuelle moyenne est de $800 \mathrm{~mm}$ avec de fortes variations spatio-temporelles. La température moyenne annuelle est de $28{ }^{\circ} \mathrm{C}$ avec des maxima mensuels de $40^{\circ} \mathrm{C}$ (de mars à avril). Le sol est de type lixisol ferrique (FAO, 2003) à texture sableuse (teneur en sables dans l'horizon de surface: $60 \%$ ).

Dispositif expérimental : L'observatoire de longue durée avait été mis en place en 1980 dans le but d'étudier l'effet de l'apport de divers MO exogènes en présence ou non de la fumure minérale sur la performance culturale d'une céréale: le Sorghum bicolor (L.) Moench. Des parcelles en monoculture de sorgho ont été soumises à des apports ou non de fertilisants organiques Fumier de vache (10 tha-1 $\mathrm{an}^{-1}$; $1,1 \%$ de $\mathrm{N}, 19,6 \% \mathrm{C}$ ) et Paille de sorgho ( $8,3 \mathrm{tha} \mathrm{a}^{-1}$ an $1 ; 0,54 \% \mathrm{~N}$ et $42,0 \% \mathrm{C}$ ) et minéraux (Urée à $60 \mathrm{~kg} \mathrm{~N}$. $\mathrm{ha}^{-1} \mathrm{an}^{-1}$ ). Le dispositif est un plan agronomique en 6 blocs randomisés comportant 6 traitements (Villenave et al., 2010): des agrégats. Nous avons montré récemment en conditions contrôlée de laboratoire que dans les sols sableux du centre du Sénégal, la formation et la stabilité des macroagrégats sont assurées principalement par les activités fongiques (Sall et al. 2016). Ces résultats, obtenus avec des incubations de courte durée, révèlent une prédominance de l'activité fongique sur les hyphes mycéliens dans la stabilité des agrégats des sols sableux tropicaux. II est nécessaire d'étudier ces relations dans certains sols sableux très peu structurés de l'Afrique de l'Ouest. L'objectif de ce travail a été d'étudier l'effet d'une application sur le long terme d'intrants organiques et minéraux sur la formation d'agrégats stables et les activités microbiennes d'un sol tropical sableux du Burkina Faso.

- $\quad$ Sol Témoin sans fertilisant organique (T) et le sol avec urée $(\mathrm{T}+\mathrm{N})$

- $\quad$ Sol amendé avec la Paille $(P)$ et le sol avec apport d'urée $(\mathrm{P}+\mathrm{N})$

- $\quad$ Sol amendé avec du Fumier (F) et le sol avec apport d'urée $(\mathrm{F}+\mathrm{N})$

Le rendement en grains ainsi que la production de biomasse par $\mathrm{m}^{2}$ obtenus au moment des prélèvements de sols sont dans le tableau 1.

Prélèvement de sols : En Octobre 2007, à la fin du cycle cultural, sur chaque parcelle élémentaires ( 36 parcelles au total), trois échantillons de sol de $1000 \mathrm{~cm}^{3}$ $(10 \mathrm{~cm} \times 10 \mathrm{~cm} \times 10 \mathrm{~cm})$ ont été prélevés sur l'horizon $0-10 \mathrm{~cm}$ à l'aide de cubes métalliques enfoncés avec précaution pour perturber le moins possible la structure du sol en place. Chaque cube de sol ainsi obtenu a été subdivisé en deux sous-échantillons pour les analyses. Un sous échantillon, gardé à $+4^{\circ} \mathrm{C}$ sera utilisé pour la séparation des classes d'agrégats et les analyses microbiologiques. Un sous-échantillon, séché à température ordinaire a été utilisé pour les analyses physico-chimiques (Villenave et al., 2010). Les quantités totales de carbone (C), d'azote (N) et de phosphore sont répertoriées dans le tableau 1. 
Tableau 1 : Effet des fertilisants organiques $(F O)$ et minéraux $(N)$ sur les teneurs en $C, N$ et $P$ du sol et sur les rendements du sorgho en tha-1.

\begin{tabular}{|c|c|c|c|c|c|}
\hline & C total & $\mathrm{N}$ total & $P$ total & Grains & nasse \\
\hline & \multicolumn{3}{|c|}{$\mathrm{g} \mathrm{kg}^{-1}$} & \multicolumn{2}{|c|}{$\mathrm{T} \mathrm{ha}^{-1}$} \\
\hline Témoin (T) & A 2,16 a & $\mathrm{A} 0,13 \mathrm{a}$ & A $0,11 \mathrm{a}$ & A 0,43 a & $A B 1,93$ a \\
\hline $\mathrm{T}+\mathrm{N}$ (urée) & $2,22 \mathrm{a}$ & $0,14 a$ & $0,97 \mathrm{a}$ & 0,67 a & $2,81 \mathrm{a}$ \\
\hline Pailles (P) & A $2,48 \mathrm{a}$ & $A 0,13 a$ & A 0,11 a & $A B \quad 0,69 a$ & A 1,72 a \\
\hline $\mathrm{P}+\mathrm{N}$ (urée) & $2,69 a$ & $0,15 a$ & $0,11 a$ & $0,78 a$ & $3,42 \mathrm{~b}$ \\
\hline Fumier (F) & в 3,56 a & в $0,27 \mathrm{a}$ & в 0,15 a & B 1,31 a & в 3,37 a \\
\hline $\mathrm{F}+\mathrm{N}$ (urée) & $3,57 \mathrm{a}$ & $0,25 a$ & $0,15 a$ & $1,46 \mathrm{a}$ & $3,77 \mathrm{a}$ \\
\hline Anova FO (a) & $* * *$ & $\star * *$ & ** & ** & * \\
\hline Anova N & ns & ns & ns & ns & * \\
\hline Anova FO x N & ns & ns & ns & ns & ns \\
\hline
\end{tabular}

Les lettres capitales comparent les différences entre traitements (Témoin, Paille, Fumier), et les lettres minuscules comparent les différences entre apport ou non de fertilisation azotée $(\mathrm{N}=$ urée). La significativité est déterminée au seuil de 0.05 (Test de Fisher's LSD). $n=6$.

(a) Interaction entre les fertilisants organiques (FO) : Anova des valeurs de F significatif à : ${ }^{*} p<0,05 ;{ }^{* \star} p<0,01 ;{ }^{* \star *} p<0,001$; ns : non significatif.

\section{Analyses}

Séparation des classes d'agrégats: La distibution des classes d'agrégats formés a été mesurée par tamisage à l'eau par une série de trois mailles de tamis (2, 0,25 et $0,05 \mathrm{~mm})$ selon la méthode adaptée d'Elliott (1986). Quatres classes d'agrégats ont été isolées : > $2000 \mu \mathrm{m}$ (macro-agrégats), 250-2000 $\mu \mathrm{m}$ (mésoagrégats), $50-250 \mu \mathrm{m}$ (microagrégats) et la fraction fine $<50 \mu \mathrm{m}$ (limono-argileuse). Brièvement, sur un tamis de $2 \mathrm{~mm}$ un échantillon de $100 \mathrm{~g}$ de sol a été préalablement immergé pendant 5 minutes dans de l'eau déminéralisée. Pendant le tamisage, les agrégats du sol ont été séparés en secouant verticalement (amplitude de $3 \mathrm{~cm}$ ) 50 fois le tamis pendant 2 minutes. Les classes d'agrégats séparés ont été récupérées dans des coupelles préalablement tarées, séchées et pesées (Sall et al., 2016). Le Diamètre Moyen Pondéré (DMP) ou Mean Weigth Diameter (MWD) a été calculée suivant la méthode de van Bavel (1950). Le DMP a été utilisé comme un indicateur de la stabilité des agrégats. $\mathrm{DMP}=((2 \times[\%>2 \mathrm{~mm}])+(1,125 \times[\% 0,25-2 \mathrm{~mm}])+$ $(0,15 \times[\% 0,05-0,25 \mathrm{~mm}])+(0,05 \times[\%<0,05 \mathrm{~mm}])) /$ 100. Les coefficients $2 ; 1,125 ; 0,15 ; 0,05$ sont les diamètres moyens entre les mailles respectives des tamis $>2,2$ et $0,25 \mathrm{~mm}, 0,25$ et $0,05 \mathrm{~mm}, 0,05$ et 0 $\mathrm{mm}$.

Potentiel de respiration du sol: Le potentiel de respiration du sol a été déterminé en incubant $10 \mathrm{~g} \mathrm{du}$ sol total non fractionné, humidifié à $100 \%$ de sa capacité de rétention (8 g d'eau pour $100 \mathrm{~g}$ de sol) dans un flacon à $28^{\circ} \mathrm{C}$ pendant 7 jours. Les mesures de $\mathrm{CO}_{2}$ ont été effectuées tous les jours par injection directe du gaz dans un chromatographe à phase gazeuse (Sall et al., 2016).

Mesure des activités enzymatiques: L'activité chitinase ( $\mathrm{N}$ - acétyl- $\beta$-glucosamidase) a été déterminée par la méthode adaptée de Hayano (1973).

Mesure de la longueur des hyphes mycéliens: L'extraction des hyphes mycéliens a été déterminée sur selon la méthode décrite par Hassen et al. (1974). La longueur totale du mycélium exprimé en $\mathrm{m} \mathrm{g}^{-1} \mathrm{sol} \mathrm{sec}$, a été mesurée par la technique du "Gridline intersection method » comme décrit par Malcová et al. (2002).

Traitements statistiques: L'effet des différents facteurs sur les paramètres étudiés a été testé par analyse de variance à un facteur et à deux facteurs à l'aide du logiciel XLSTAT (Addinsoft version 2008.7.03). La différence entre les moyennes des traitements a été évalué par un test de comparaison des moyennes avec le test de Fisher's LSD $(P<0,05)$. Des régressions linéaires entre les fertilisants organiques (FO : Fumier et Paille) et/ou minéraux ( $\mathrm{N}$ : Urée) ont été également calculées et leur importance (Anova des valeurs de F) a été notée ns pour $p \geq 0,05,{ }^{*}$ pour $p<0,05$, ${ }^{* *}$ pour $p$ $<0,01$ et ${ }^{* * *}$ pour $p<0,001$. Enfin, une Analyse en 
Composante Principale (ACP) a été également réalisée avec le même logiciel pour classer les traitements selon

\section{RESULTATS}

Effet sur les propriétés chimiques du sol et la productivité végétale : Le tableau 1 montre un effet significatif des apports de fertilisants organiques. Les teneurs en $\mathrm{C}, \mathrm{N}$ et $\mathrm{P}$ sont significativement plus élevées dans le sol amendé avec le fumier que dans le sol amendé avec la paille. Aucun effet significatif de la fertilisation minérale n'a été noté sur ces teneurs. Le sol amendé au fumier pendant plusieurs années entraine un rendement en grains significativement plus élevé que le sol témoin (non amendé) et un rendement en biomasse végétale significativement plus élevé que le sol amendé avec la paille. II apparaît aussi un effet significatif de l'apport de l'urée sur la biomasse de la paille. Toutefois, pour tous les paramètres mesurés, aucune interaction significative n'a été obtenue entre la fertilisation organique (FO) et la fertilisation minérale (N).

Effet des fertilisants organiques et minéraux sur la formation des agrégats : Pour chaque traitement, le poids total des classes d'agrégats isolés représentait 98 à 103\% du poids du sol non fractionné (Tableau 2).

Quel que soit le type d'intrant organique apporté, les les variables mesurées.

micro-agrégats $(50-250 \mu \mathrm{m})$ sont dominants comparés aux autres classes d'agrégats. Ils représentent respectivement $53 \%$ pour le sol témoin et $49 \%$ pour les sols amendés avec les résidus organiques. Les macroagrégats $(>2000 \mu \mathrm{m})$ représentent de petite quantité avec $0,2,1,6$ et $1,7 \%$ respectivement le sol témoin, le sol amendé avec la paille et le sol amendé avec le fumier. Les apports de résidu à long terme ont entrainé une diminution sensible des micro-agrégats et une augmentation significative des macro-agrégats comparés au sol témoin. Cependant, aucune différence significative n'est notée entre les traitements de résidus. Le facteur apport organique a été significatif sur le paramètre macroagrégats $>2000 \mu \mathrm{m}$ contrairement aux autres classes d'agrégats. La fertilisation minérale a un effet significativement négatif sur la formation des macroagrégats $>2000 \mu \mathrm{m}$ du sol amendé avec les résidus de Fumier (Tableau 2). Par contre, cette fertilisation azotée n'a pas eu d'effet significatif sur les traitements Témoin et Paille.

Tableau 2 : Effet de la qualité des intrants organiques sur la distribution des différentes tailles d'agrégats isolées.

\begin{tabular}{|c|c|c|c|c|c|}
\hline & $\mathrm{Ag}>2000 \mu \mathrm{m}$ & $250-2000 \mu \mathrm{m}$ & $50-250 \mu \mathrm{m}$ & $<50 \mu \mathrm{m}$ & \multirow{2}{*}{$\frac{\mathrm{DMP}^{(\mathrm{b})}}{\mathrm{cm}}$} \\
\hline & \multicolumn{4}{|c|}{$\%$ sol } & \\
\hline Témoin (T) & $\mathrm{A} 0,204 \mathrm{a}$ & A $26,631 \mathrm{a}$ & A 53,079 a & A 19,211 a & ${ }^{\mathrm{A}} 0,39 \mathrm{a}$ \\
\hline $\mathrm{T}+\mathrm{N}$ (urée) & $0,199 a$ & $25,362 \mathrm{a}$ & $54,7 \mathrm{a}$ & $19,651 \mathrm{a}$ & $0,38 a$ \\
\hline Pailles (P) & $A B 1,611 a$ & A 28,301 a & A 49,405 a & A 20,967 a & A $0,44 \mathrm{a}$ \\
\hline $\mathrm{P}+\mathrm{N}$ (urée) & $0,882 a$ & $27,234 \mathrm{a}$ & $50,120 a$ & $21,025 \mathrm{a}$ & $0,41 a$ \\
\hline Fumier (F) & в $1,760 \mathrm{~b}$ & A 27,820 a & A 49,437 a & A 19,978 a & A $0,43 a$ \\
\hline $\mathrm{F}+\mathrm{N}$ (urée) & $0,511 \mathrm{a}$ & 29,126 a & $50,965 \mathrm{a}$ & $19,123 \mathrm{a}$ & $0,42 \mathrm{a}$ \\
\hline Anova FO (a) & $* * *$ & ns & ns & ns & ns \\
\hline Anova N & ns & ns & ns & ns & ns \\
\hline Anova FO x N & * & ns & ns & ns & ns \\
\hline
\end{tabular}

Les lettres capitales comparent les différences entre traitements (Témoin, Paille, Fumier), et les lettres minuscules comparent les différences entre apport ou non de fertilisation azotée $(\mathrm{N}=$ urée). La significativité est déterminée au seuil de 0.05 (Test de Fisher's LSD). $n=6$.

(a) Interaction entre les fertilisants organiques (FO) : Anova des valeurs de F significatif à : ${ }^{*} p<0,05 ;{ }^{* \star} p<0,01 ;{ }^{* \star *} p<0,001$; ns : non significatif.

(b) $\mathrm{DMP}=$ Diamètre moyen Pondéré (DMP). 
Effet des fertilisants organiques et minéraux sur les propriétés microbiennes du sol : L'amendement à long terme a montré un effet significatif sur l'activité enzymatique de la chitinase et sur l'activité respiratoire (Tableau 3). L'activité chitinase est significativement plus élevée dans les traitements Fumier et sol témoin. L'amendement avec le Fumier présente une activité respiratoire du sol significativement la plus élevée (117,3 $\left.\mathrm{mg} \mathrm{C}^{-} \mathrm{CO}_{2} \mathrm{~kg}^{-1} \mathrm{sol}\right)$ et le sol témoin présente l'activité la plus faible $\left(64,6 \mathrm{mg} \mathrm{C}-\mathrm{CO}_{2} \mathrm{~kg}^{-1} \mathrm{sol}\right)$.La longueur des hyphes est plus élevée dans le traitement avec le Fumier, mais la différence n'est pas significative. Aucun effet significatif des amendements organiques n'a été trouvé sur la mesure des hyphes fongiques. La fertilisation azotée n'a pas eu d'effet significatif sur les propriétés microbiennes du sol mesurées (Tableau 3).

Tableau 3 : Effet des fertilisants organiques et minéraux sur les propriétés microbiennes (chitinase et respiration du sol) et la longueur des hyphes fongiques du sol non fractionné

\begin{tabular}{|c|c|c|c|}
\hline & \multirow{2}{*}{$\begin{array}{l}\text { Chitinase } \\
{\mathrm{mg} \mathrm{pNPP} . \mathrm{kg}^{-1} \text { sol.h }^{-1}}\end{array}$} & \multirow{2}{*}{$\begin{array}{l}\mathrm{CO}_{2} \\
\mathrm{mg} \mathrm{C}-\mathrm{CO}_{2} \cdot \mathrm{kg}^{-1} \mathrm{sol}\end{array}$} & \multirow{2}{*}{$\begin{array}{l}\text { Hyphes } \\
\mathrm{m} \cdot \mathrm{g}^{-1} \mathrm{sol} \\
\end{array}$} \\
\hline & & & \\
\hline Témoin (T) & в 44,83 a & A $64,6 \mathrm{a}$ & ${ }^{A} 0,30 a$ \\
\hline T+N (urée) & $39,12 \mathrm{a}$ & $54,7 \mathrm{a}$ & $0,21 \mathrm{a}$ \\
\hline Pailles (P) & A 37,19 a & в 90,8 a & A 0,32 a \\
\hline $\mathrm{P}+\mathrm{N}$ (urée) & $38,79 a$ & 93,3 a & $0,41 \mathrm{a}$ \\
\hline Fumier $(\mathrm{F})$ & в 45,63 a & c 117,3 a & A $0,39 a$ \\
\hline $\mathrm{F}+\mathrm{N}$ (urée) & $45,51 \mathrm{a}$ & $114,9 a$ & $0,25 \mathrm{a}$ \\
\hline Anova FO & * & $* * \star$ & ns \\
\hline Anova N & ns & ns & ns \\
\hline Anova FO x N & ns & ns & ns \\
\hline
\end{tabular}

Les lettres capitales comparent les différences entre traitements (Témoin, Paille, Fumier), et les lettres minuscules comparent les différences entre apport ou non de fertilisation azotée $(\mathrm{N}=$ urée). La significativité est déterminée au seuil de 0.05 (Test de Fisher's LSD). $n=6$.

(a) Interaction entre les fertilisants organiques (FO) : Anova des valeurs de F significatif à : ${ }^{*} p<0,05 ;{ }^{* *} p<0,01 ;{ }^{* * *} p<0,001$; ns : non significatif.

Relation entre l'agrégation et les propriétés microbiennes : L'Analyse en Composante Principale (ACP) a permis de comparer les agrégats, les paramètres chimiques et les propriétés microbiennes du sol (Fig. 1A et 1B). Les deux axes du plan factoriel ont représenté environ $89 \%$ de l'inertie totale. L'axe F1 qui représenté $61 \%$ de la variance, sépare le sol témoin avec les sols amendés avec les résidus. Cela est expliqué par les micro-agrégats $(50-250 \mu \mathrm{m})$ plus élevés dans le sol témoin et les meso et macroagrégats plus élevés dans les sols amendés avec les résidus. L'axe F2 qui représente $28 \%$ de la variance, sépare les deux sols amendés avec les résidus organiques (Fig. 1A). L'ACP a indiqué une corrélation directe entre les macro-agrégats $>2000 \mu \mathrm{m}$ et le DMP $(r=0,88)$ et avec l'activité respiratoire $(r=0,64)$ (Fig. 1B). Les micro-agrégats $(50-250 \mu \mathrm{m})$ sont inversement corrélés aux macro-agrégats et à l'activité respiratoire $(r$ $=-0,87$ et $r=-0,84$ respectivement). Aucune corrélation n'a été trouvée entre l'activité de la chitinase et les agrégats des sols amendés à long terme. II y'a une corrélation positive entre la formation des macroagrégats $(\mathrm{Ag}>2000 \mu \mathrm{m})$ et les hyphes fongiques ( $r=$ $0,66)$. 


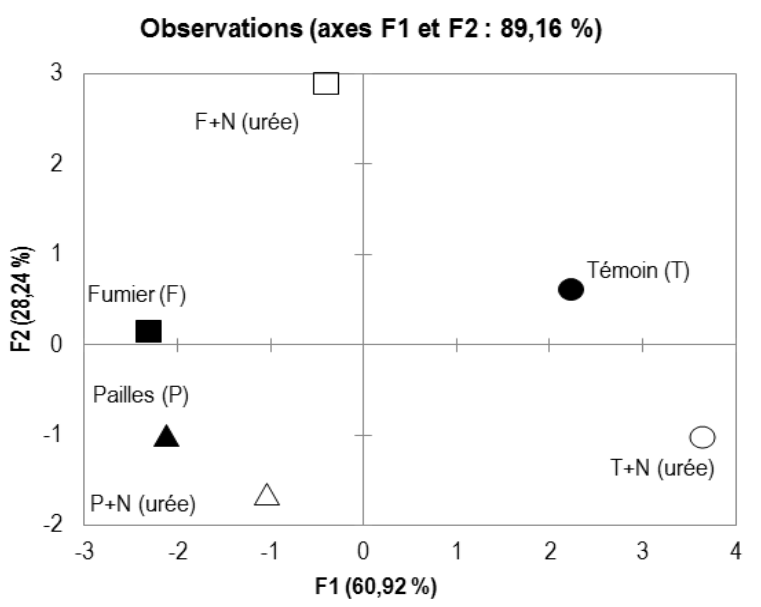

A)

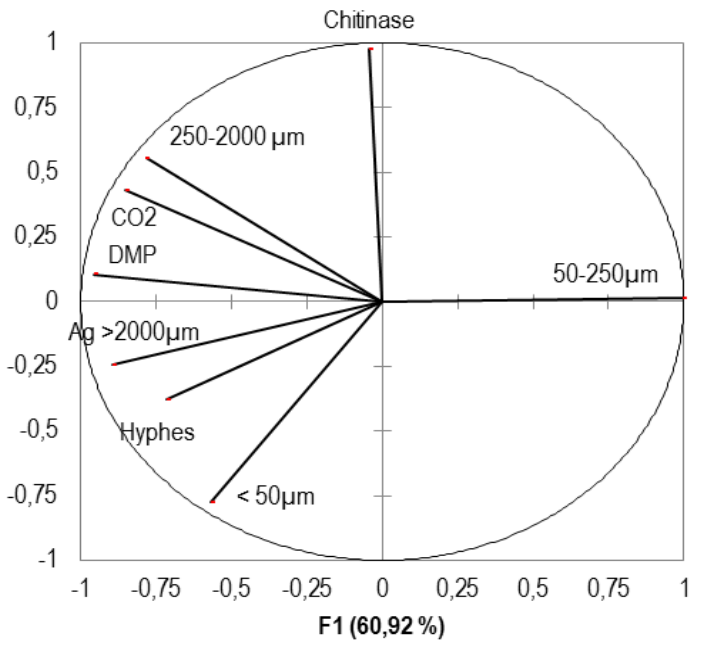

B)

Figure 1 : Analyse en Composante Principale (ACP) constitué par le cercle de corrélation associé $(A)$ le plan factoriel (B) et comparant les données $\mathrm{Ag}>2000 \mu \mathrm{m}$, les propriétés microbiennes du sol total (Hyphes fongiques, chitinase, activité respiratoire). Témoin $(T)$, Paille $(P)$, Fumier $(F)$, Urée $=(+N) ; n=6$

\section{DISCUSSION}

Effet des fertilisants organiques sur la formation des agrégats: Comparé au sol témoin, les sols amendés avec les fertilisants organiques augmentent la quantité des macro-agrégats. Cette augmentation est due à la stimulation de la biomasse et des activités microbienne dans ce site expérimental (Diallo-Diagne et al., 2016) lesquelles sont à l'origine de la formation des agrégats (Abiven et al., 2009). Certains travaux ont montré que l'agrégation et la stabilité structurale sont positivement corrélées avec la qualité et la quantité de MO incorporée au sol (Martens, 2000; Abiven et al., 2007 ; Chivenge et al., 2011). Cependant, nos résultats n'ont pas montré une relation significative entre le type d'intrants organiques apportés (Fumier vs Paille) et la formation des macroagrégats > $2000 \mu \mathrm{m}$. Ils corroborent nos résultats obtenus pour des incubations effectuées sur une courte période avec des résidus de Maïs et de Crotalaire (Sall et al., 2016). D'autres auteurs ont également conclu récemment à une absence de relation entre la qualité des fertilisants et la stabilité des agrégats (Fonte et al., 2010; Gentile et al., 2010). Nous pensons que la différence de qualité biochimique de nos résidus pourrait induire une vitesse de minéralisation initiale différente, donc une relation significative avec la formation des agrégats à moyen terme (sur quelques mois). Mais cette différence sur la décomposabilité des résidus disparaissant au cours du temps, et la stabilisation du $\mathrm{C}$ à long terme devient équivalente. Un suivi de l'agrégation tous les mois au cours de la décomposition et un dosage du $C$ dans les agrégats pourraient permettre de tirer une conclusion définitive. De plus, le labour qui est effectué tous les ans depuis 27 ans sur les parcelles (Mando et al., 2005) entraine une destruction des macroagrégats > $2000 \mu \mathrm{m}$ pour former les mesoagrégats et microagrégat $(250-2000 \mu \mathrm{m}$ et $50-250 \mu \mathrm{m})$. Plusieurs auteurs ont souligné l'effet destructif du labour sur les agrégats en particulier sur les macro-agrégats (Ouattara et al., 2008 ; Curaqueo et al., 2010). Zida (2011) a montré sur cette même station, que toutes les pratiques culturales (apport d'intrants organo-minéraux, rotation culturale, labour et grattage du sol) ont eu un effet négative sur la macro-agrégation du sol $(2-8 \mathrm{~mm})$ comparé à un sol en jachère.

Les propriétés microbiennes et l'agrégation du sol : Le potentiel de respiration $\mathrm{CO}_{2}$ est significativement plus élevé dans le sol amendé avec le Fumier comparé au sol amendé avec la Paille et le sol non amendé. La décomposabilité est plus élevée dans le sol amendé avec Fumier. Le potentiel de respiration est positivement corrélé aux macro-agrégats $>2000$ $\mu \mathrm{m}(r=0,64)$ et aux méso-agrégats $(r=0,86)$. Ce résultat suggère que la différence sur la décomposabilité des résidus organiques induit dans le long terme une différence significative dans les mésoagrégats $(250-2000 \mu \mathrm{m})$. Bronick \& Lal (2005) ont 
montré que l'augmentation dans la minéralisation du $\mathrm{C}$ est associée à une augmentation de la stabilité des macro- et méso-agrégats.

La mesure de la chitinase a été longtemps considérée comme étant un indicateur indirect de l'activité et la présence des populations fongiques (Miller et al., 1998). Le rôle des activités fongiques dans la formation et la stabilisation des agrégats a été démontré en utilisant la chitinase dans une expérimentation sur une courte période de 4 mois (Sall et al., 2016). Ces travaux suggèrent que l'activité chitinase, qui reflète l'activité de la biomasse fongique, est l'indicateur le plus pertinent associé à l'agrégation des sols sableux. Cependant, les résultats de cette étude à long terme montrent qu'il n'existe aucune relation directe entre les macroagrégats > $2000 \mu \mathrm{m}$, et l'activité chitinase. Cette contradiction peut être expliquée par l'effet destructeur des champignons (hyphes et activités) par le labour annuel (Janusauskaite et al., 2013, Zhang et al., 2014). II semble que cette relation entre les activités fongiques et l'agrégation soit plus complexe en situation réelle, au champ. En effet, d'autres facteurs agrégeants du sol peuvent également entrer en jeu sous l'action de la nématofaune et des effets rhizosphèriques environnants (Moreno-Espíndola et al., 2007). Concernant ces derniers, nous avons trouvé une corrélation positive $(r=0,68)$ entre les macroagrégats larges et les hyphes fongiques.

Effet de la fertilisation minérale : Un effet significativement négatif de la fertilisation azotée a été observé dans les macroagrégats $>2000 \mu \mathrm{m}$ pour les traitements Fumier. Bossuyt et al. (2001) ont notée également un effet négatif de la fumure minérale sur les macroagrégats au cours d'une incubation de 14 jours.

\section{CONCLUSIONS}

Les résultats de l'application sur le long terme des résidus organiques ont montré une augmentation de la quantité des macro-agrégats (> $2000 \mu \mathrm{m})$. Cependant, aucune relation significative n'a été obtenue avec la qualité des résidus organiques. II suit que la décomposabilité des résidus organiques induirait dans le long terme une différence significative dans les méso-agrégats $(250-2000 \mu \mathrm{m})$. Notre étude a montré que la mesure des hyphes fongiques semble plus déterminante dans la formation des macro-agrégats à long terme, par opposition aux activités fongiques dans le court terme. L'apport d'azote minéral exogène
Pourtant, nous n'avons pas trouvé d'effet significatif de l'apport exogène d'azote minéral sur la formation des macro-agrégats pour des incubations de 4 mois avec des résidus de Maïs et de Crotalaria (Sall et al., 2016). Par ailleurs, certains auteurs considèrent que l'azote minéral exogène diminue l'activité microbienne et par conséquent inhibe la production de métabolites microbiens qui sont les agents agrégeants responsables de la formation des macro-agrégats (Bossuyt et al., 2001; Chivenge et al., 2011). Nos résultats n'ont pas montré d'effet significatif de l'azote minéral sur les paramètres microbiologiques mesurés. \|l en est de même des teneurs en $C, N$ et $P$. II semble probable que dans le long terme, l'effet différencié de l'azote minéral exogène doit être cherché dans la qualité intrinsèque des résidus en décomposition. En effet, la qualité biochimique des résidus en décomposition ( $\mathrm{N}$, lignine et polyphénols), peut influer sur les macro-agrégats (Six et al., 2001, Chivenge et al., 2011). Par exemple, il a été démontré une corrélation positive de l'agrégation avec la concentration en polyphénols (Griffiths \& Burns, 1972; Martens, 2002). Cependant, l'azote minéral exogène pourrait complexer les polyphénols et les empêcher de participer à la formation des agrégats (Sall et al., 2003). Dans nos travaux, nous avons une réduction d'au moins de moitié des macro-agrégats pour les traitements résidus avec $\mathrm{N}$ minéral. Cette réduction est significativement plus élevée pour le traitement Fumier qui pourrait contenir plus de polyphénols que la Paille. Un dosage des polyphénols dans ces résidus et dans les macro-agrégats nous aurait permis de valider cette hypothèse.

entraine une diminution de la macro-agrégation. Les résultats suggèrent que cet effet négatif ne résulte pas d'une inhibition de l'activité fongique, mais d'une complexation des polyphénols, agents agrégeants importants dans le processus d'agrégation pour le long terme. Le labour annuel pourrait également influencer la formation des agrégats. II serait souhaitable de valider l'importance des polyphénols dans l'agrégation des sols tropicaux sableux en menant des expérimentations sur le long terme sans labourer les parcelles. 


\section{REMERCIEMENTS}

Ce travail a été possible grâce au soutien financier de l'Agence Universitaire de la Francophonie (AUF). Nos remerciements aux techniciens de la station Saria du Burkina Faso et du Laboratoire d'Ecologie Microbienne

\section{BIBLIOGRAPHIE}

Abiven S, Menasseri S, Chenu C, 2009. The effects of organic inputs over time on soil aggregate stability - A literature analysis. Soil Biology and Biochemistry 41: 1-12.

Abiven S, Menasseri S, Leterme P, 2007. Dynamics of aggregate stability and biological binding agents during the decomposition of organic material. European Journal of Soil Science 58: 239-247.

Bationo A, Kihara J, Kimetu J, 2007. Soil organic carbon dynamics, functions and management in West African agro-ecosystems. Agricultural Systems 94: 13-25.

Berg B and Matzner E, 1997. Effect of $\mathrm{N}$ deposition on decomposition of plant litter and soil organic matter in forest ecosystems, Environmental Reviews 5: 1-25.

Bossuyt H, Denef K, Six J, Paustian K, 2001. Influence of microbial populations and residue quality on aggregate stability. Applied Soil Ecology 16: 195-208.

Bremer E, Van Houtoum W, Kessel C, 1991. Carbon dioxide evolution from wheat and lentil residues as affected by grinding, added nitrogen and the absence of soil Biology and Fertility of Soils 11: 221-227.

Bronick, CJ and Lal R, 2005. Soil structure and management: a review. Geoderma 124: 3-22.

Chivenge P, Vanlauwe B, Gentile R, Six J, 2011. Comparison of organic versus mineral resource effects on short-term aggregate carbon and nitrogen dynamics in a sandy soil versus a fine textured soil, Agriculture Ecosystem and Environment 140: 361-371.

Curaqueo G, Acevedo E, Borie F, 2010. Tillage effect on soil organic matter, mycorrhizal hyphae and aggregates in a Mediterranean agroecosystem.. Journal of Soil Science and Plant Nutrition $10: 12-21$.

Diallo-Diagne NH, Assigbetse K, Sall SN, Masse D, Bonzi M, Ndoye I, Chotte JL, 2016. Response of Soil Microbial Properties to Long-Term Application of Organic and Inorganic Amendments in a Tropical Soil (Saria, Burkina des Sols et Agrosystèmes Tropicaux (LEMSAT) du LMI IESOL pour les analyses des sols. Nous remercions également les corrections apportées par les relecteurs.

Faso). Open Journal of Soil Science6, 21-33. http://dx.doi.org/10.4236/ojss.2016.62003

Donnison LM, Griffith GS, Bardgett RD, 2000. Determinants of fungal growth and activity in botanically diverse hay meadows: effects of litter type and fertilizer additions Soil Biology and Biochemistry 32: 289-294.

Elliott ET, 1986. Aggregate structure and carbon, nitrogen, and phosphorus in native and cultivated soils. Soil Science Society of America Journal 50: 627-633.

FAO, 2003. World reference base for soil resources. World soil resources reports, Food and Agricultural Organization, Rome.

Fonte SJ, Yeboah, E, Six J, 2010. Fertilizer and residue quality effects on organic matter stabilization in soil aggregates. Soil Science Society of America Journal. 73: 961-966.

Gentile R, Vanlauwe B, Kavoo A, Chivenge P, Six J, 2010. Residue quality and $N$ fertilizer do not influence aggregate stabilization of $\mathrm{C}$ and $\mathrm{N}$ in two tropical soils with contrasting texture. Nutrient Cycling and Agroecosystem 88: 121131.

Griffiths E, Burn R, 1972. Interaction between phenolic substances and microbial polysaccharides in soil aggregation. Plant and Soil 36, 599-612.

Hanssen JF, Thingstad TF, Goksoyr J, 1974. Evaluation of hyphal lengths and fungal biomass in soil by a membrane filter technique Oikos 25: 102-107.

Hayano K, 1973. A method for determination of $\beta$ glucosidase activity in soil. Soil Science and Plant Nutrition 19: 103-108.

Janušauskaite D, Kadžienè G, Auškalnienè O, 2013. The Effect of Tillage System on Soil Microbiota in Relation to Soil Structure. Pollution Journal of Environmental Studies Vol. 22, No. 5 : 1387-1391

Martens DA, 2000. Plant residue biochemistry regulates soil carbon cycling and carbon sequestration. Soil Biology and Biochemistry 32: 361-369.

Martens DA, 2002. Relationship between plant phenolic acids released during soil mineralization and 
aggregate stabilization. Soil Science Society of America Journal 66: 1857-1867.

Malcová R, Gryndler M, Vosátka M, 2002. Magnesium ions alleviate the negative effect of manganese on Glomus claroideum BEG23. Mycorrhiza 12: 125-129.

Mando A, Ouattara B, Vanlauwe B, 2005. Long-term effect of tillage and manure application on soil organic fractions and crop performance under Sudano-Sahelian conditions. Soil \& Tillage Research 80 : 95-101.

Miller M, Palojärvi A, Rangger A, KjØller A, 1998. The use of fluorogenic substrates to measure fungal presence and activity in Soil Applied Environmental of Microbiology 64: 613-617.

Moreno-Espíndola IP, Rivera-Becerril F, De LeónGonzález F, 2007. Role of root-hairs and hyphae in adhesion of sand particles. Soil Biology and Biochemistry 39: 2520-2526.

Ouattara K, Ouattara B, Malmer A, 2008. Effects of ploughing frequency and compost on soil aggregate stability in a Cotton-Maize (Gossypiumhirsutum-zea mays L.) rotation system in Burkina Faso. Soil Use and Management 24: 19-28.

Ouattara K, Ouattara Badiori O, Sédogo PM, 2006. Long-term effect of ploughing, and organic matter input on soil moisture characteristics of a Ferric Lixisol in Burkina Faso. Soil Tillage Research 88: 217-224.

Rillig MC, Mardatin F, Antunes M, 2010. Mycelium of arbuscular mycorrhizal fungi increases soil water repellency and is sufficient to maintain water-stable soil aggregates. Soil Biology and Biochemistry 42: 1189-1191.

Sall SN, Masse D, Bernhard-Reversat F, Guissé A, Chotte JL, 2003. Microbial activities during the early stage of laboratory decomposition of tropical leaf litters: the effect of interactions between litter quality and exogeneous inorganic nitrogen Biology Fertility of Soils 39: 103-111.

Sall SN, Masse D, Diallo NH, Sow TMB, Hien E, Guisse A, 2016. Effects of residue quality and soil mineral $\mathrm{N}$ on microbial activities and soil aggregation in a tropical sandy soil in Senegal. European Journal of Soil Biology 75: 62-69. dx.doi.org/10.1016/j.ejsobi.2016.04.009

Six J, Feller C, Denef K, Albrecht A, 2002. Organic matter, biota and aggregation in temperate and tropical soils: effects of no-tillage.
Agronomie: Agriculture and Environment 22: 755- 775.

Six J, Elliot ET, Paustian K, 2000. Soil macroaggregate turnover and microaggregate formation: a mechanism for $\mathrm{C}$ sequestration under notillage agriculture. Soil Biology and Biochemistry 32: 2099-2103.

Tisdall JM \& Oades JM, 1982. Organic matter and water-stable aggregates in soil. Journal of Soil Science 33, 141-163.

Van Bavel CHM, 1950. Mean-weight diameter of soil aggregates as a statistical index of aggregation. 14: 20-23.

Villenave C, Saj S, Pablo AL, Sall SN, Djigal D, Chotte JL, Bonzi M., 2010. Influence of long-term organic and mineral fertilization on soil nematofauna when growing Sorghum bicolor in Burkina Faso. Biology Fertility of Soils 46: 659-670.

Zhang B, Li Y, Ren T et al. 2014. Short-term effect of tillage and crop rotation on microbial community structure and enzyme activities of a clay loam soil. Biology Fertility of Soils 50: 1077. doi:10.1007/s00374-014-0929-4

Zida Z, 2011. Long-term effects of conservation soil management in Saria, Burkina Faso, West Africa. Thesis, Wageningen University, Wageningen, NL. 142p. 